\title{
Study boundary problem with Integral condition for Fractional Differential Equations
}

\author{
Nawal A. Abdulkader ${ }^{*}$, Nadia A. Abdul Razaq ${ }^{2}$ \\ ${ }^{1,2}$ Department of Mathematics, College of Education for Pure Science, University of Mosul, Mosul, Iraq \\ E-mail: ${ }^{1 *}$ nawalazizmath@gmail.com, ${ }^{2}$ nadiaadnan@uomosul.edu.iq
}

(Received January 07, 2020; Accepted April 27, 2020; Available online September 01, 2020)

DOI: 10.33899/edusj.2020.126471.1038, () 2020, College of Education for Pure Science, University of Mosul.

This is an open access article under the CC BY 4.0 license (http://creativecommons.org/licenses/by/4.0/).

\section{ABSTRACT:}

Many years ago there was a great interest in studying the existence of positive solutions for fractional differential equations. Many researchers have considered the existence of positive solutions of non-linear differential equations of non-integer order with integral boundary value conditions using fixed point theorems.

G.wang et al.(2012) investigated the following fractional differential equations ${ }^{c} D^{\alpha} \mathcal{W}(t)+f(t, \mathcal{W}(t))=0,0<t<1$ with integral boundary value condition

$$
\mathcal{W}(0)=\mathcal{W}^{\prime \prime}(0)=0, \quad \mathcal{W}(1)=\lambda \int_{0}^{1} \mathcal{W}(s) d s \quad \text { were } 2<\alpha \leq 3
$$

$\lambda$ is a positive number $(0<\lambda<2),{ }^{C} D^{\alpha}$ is the standard fractional derivative equation of Caputo who obtained his results by means of Guo-krosnosel'skii theorem in a cone also A.Cabada et at. (2013) established the following non-linear fractional differential equation with integral boundary value conditions

$D^{\alpha} \mathcal{W}(t)+f(t, \mathcal{W}(t))=0,0<t<1$

$\mathcal{W}(0)=\mathcal{W}^{\prime \prime}(0)=0, \mathcal{W}(1)=\lambda \int_{0}^{1} \mathcal{W}(s) d s$, were $2<\alpha \leq 3, \lambda>0, \lambda \neq \alpha, D^{\alpha}$ is Riemann -Liovuville standard fractional derivative. and $f$ is a continuous function. The results were based on Guokrasnosel'skii fixed point theorem in a cone .

In this paper we investigate the existent results of a positive solution for the integral boundary value conditions of the following system of equations:

$$
\begin{array}{ll}
{ }^{c} D^{\beta} h(t)+k(t, h(t))=0, & t \in(0,1) \\
h(0)=h^{\prime}(0)=h^{\prime \prime \prime}(0)=0, & h(1)=\delta \int_{0}^{1} h(n) d n
\end{array}
$$

where $3<\beta \leq 4, \delta$ is a positive number, $\delta \neq 3,{ }^{C} D^{\beta}$ denotes Caputo standard derivative and $k$ is a continuous function. Our work based on Banach's and Schauder's theorem.

Keyword: Fractional Differential Equation, Integral boundary value Conditions, Schauder's theorem, Green function.

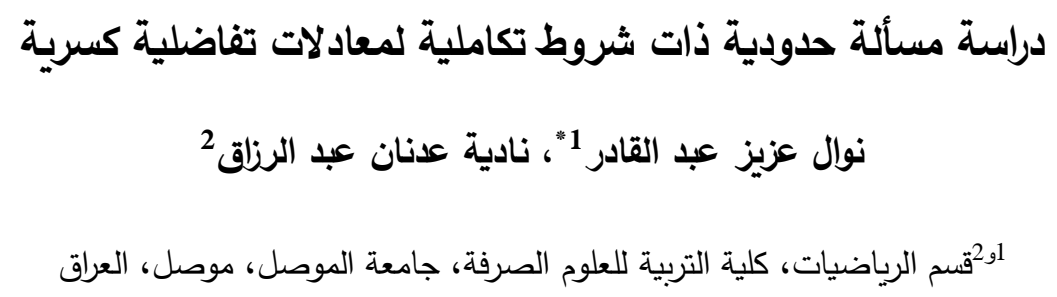


الخلاصة:

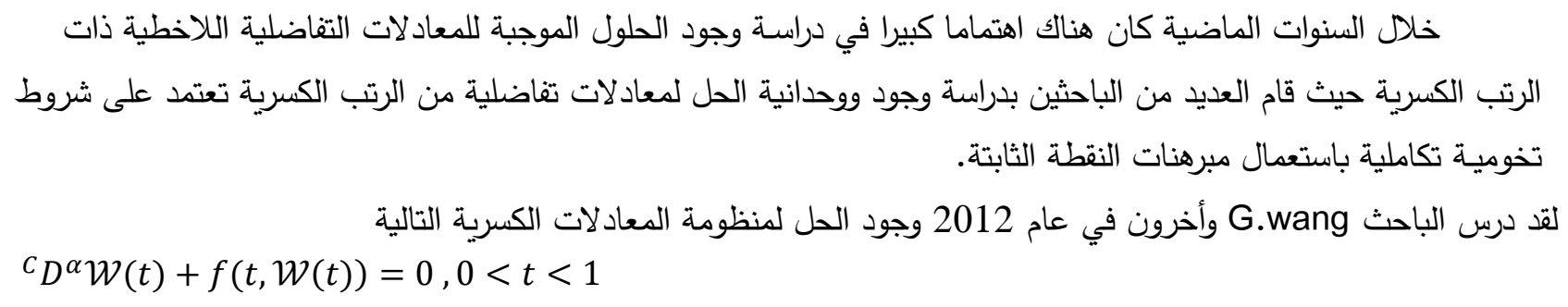

\section{Introduction}

In the last few decades, fractional order calculus has been of the most rapidly developing areas of mathematical analysis. In fact, a natural phenomenon may depend not only on the time instant but also on the previous time history, which can be successfully modeled by fractional calculus. Also fractional differential equations play an important role because of their application in various fields of science such as mathematics, physics, mechanism, economics, engineering and biological sciences etc.(see[1-4]).

Integral boundary conditions have several applications in real-life problem such as population dynamics, underground water flow, and blood flow problems. For a detailed description of the integral boundary conditions, we refer in this study to some recent papers $[5,6,7]$.

Our establishment is the existence of positive solutions of the integral boundary conditions of the following fractional differential equation:- 


$$
\begin{aligned}
{ }^{c} D^{\beta} h(t)+k(t, h(t))=0, & 0<t<1 \\
h(0)=h^{\prime}(0)=h^{\prime \prime \prime}(0)=0, & h(1)=\delta \int_{0}^{1} h(n) d n
\end{aligned}
$$

where $3<\beta \leq 4,0<\delta<3, D^{\beta}$ denotes Caputo standard derivative and $k:[0,1] \times[0, \infty) \rightarrow$ $[0, \infty)$ is a continuous function.

Firstly we obtain the exact expression of the Green's function related to the liner equation

$$
{ }^{C} D^{\beta} h(t)+g(t)=0,0<t<1
$$

corresponded to the integral condition

$$
h(0)=h^{\prime}(0)=h^{\prime \prime \prime}(0)=0, h(1)=\delta \int_{0}^{1} h(\rho) d \rho
$$

Our work is based on Banach's and Schauder's fixed point theorems.

\section{Preliminaries}

In this article, we present some definitions, notations, lemmas and theorems from fractional calculus theorem.

Definition 2.1.[1] Capote derivative fractional order $\alpha$ of the function $J:[0, \infty) \rightarrow R$ is defined as :

$$
{ }^{c} D^{\alpha} J(t)=\frac{1}{\Gamma(\rho-\alpha)} \int_{0}^{t} \frac{J^{(\rho)}(c)}{(t-c)^{1+\alpha-n}} d c, \rho=[\alpha]+1,
$$

where $[\alpha]$ is the integer part of $\alpha$.

Definition 2.2.[1] Let $q>0, g$ be a the function then the, integral of order $q$ is given by $I^{q} g(s)=\frac{1}{\Gamma(q)} \int_{0}^{s}(s-r)^{q-1} g(r) d r, q>0$

Provided the right hand exits a side.

Lemma 2.1.[8] Let $\mu>0$, the fractional differential equation ${ }^{c} D^{\mu} \mathcal{W}(t)=0$ has a unique solution given by the form

$\mathcal{W}(t)=\sum_{a=0}^{[\mu]} \frac{\mathcal{W}^{(a)}(0)}{a !} t^{a}$

Lemma 2.2. [8] let $\gamma>0$, then

$$
I^{\gamma} \quad{ }^{c} D^{\gamma} s(t)=s(t) \sum_{i=0}^{[\gamma]} \frac{s^{(i)}(0)}{i !} t^{i}
$$

Theorem 2.1.[9] (Banach contraction principle )

Let $(Y, d)$ be a complete metric space and $F: Y \rightarrow Y$ contraction. Then $F$ has a unique solution $\mathcal{W}$ and $F^{\rho}(y) \rightarrow \mathcal{W}$ for each $y \in Y$ or $(F \mathcal{W}=\mathcal{W})$.

Theorem 2.2.[9] (Schauder's fixed point theorems)

Let $Y$ be a nonempty convex subset of a locally convex liner topological space $E$, and let $\mathrm{F}: Y \rightarrow Y$ be a compact map. Then $F$ has a fixed point. 
Theorem 2.3. Let $3<\beta \leq 4$ and $\delta \neq 3$. Assume that $g \in C[0,1]$, then the problem (2)-(3) has a unique solution $h$ given by

$h(t)=\int_{0}^{1} G(t, \rho) g(\rho) d \rho$

Where

$$
G(t, \rho)=\left\{\begin{array}{l}
\frac{3 t^{2}(1-\rho)^{\beta-1}(\beta-\delta+\delta \rho)-\beta(3-\delta)(t-\rho)^{\beta-1}}{(3-\delta) \Gamma(\beta+1)}, 0 \leq \rho \leq t \leq 1 \\
\frac{3 t^{2}(1-\rho)^{\beta-1}\left(\beta-\delta+\delta_{\rho}\right)}{(3-\delta) \Gamma(\beta+1)}, 0 \leq t \leq \rho \leq 1
\end{array}\right.
$$

\section{Proof:}

According to lemma 2.2, the linear equation (3) is equivalent to the integral equation.

$h(t)=-I^{\beta} g(t)+\sum_{i=0}^{3} \frac{h^{(i)}(0)}{i !}+t^{i}$
$=-I^{\beta} g(t)+h(0)+h^{\prime}(0) t+\frac{h^{\prime \prime}(0)}{2 !} t^{2}+\frac{h^{\prime \prime \prime}(0)}{3 !} t^{3}$

Since $h(0)=h^{\prime}(0)=h^{\prime \prime \prime}(0)=0$, we get

$h(t)=-\frac{1}{\Gamma(\beta)} \int_{0}^{1}(t-\rho)^{\beta-1} g(\rho) d \rho+\frac{h^{\prime \prime}(0)}{2} t^{2}$

$h(1)=-\frac{1}{\Gamma(\beta)} \int_{0}^{1}(1-\rho)^{\beta-1} g(\rho) d \rho+\frac{h^{\prime \prime}(0)}{2}$

From condition (2), $h(1)=\delta \int_{0}^{1} h(\rho) d \rho$

we get

$h^{\prime \prime}(0)=-\frac{2}{\Gamma(\beta)} \int_{0}^{1}(1-\rho)^{\beta-1} g(\rho) d \rho+2 \delta \int_{0}^{1} h(\rho) d \rho$

Hence

$$
\begin{gathered}
h(t)=-\frac{1}{\Gamma(\beta)} \int_{0}^{t}(t-\rho)^{\beta-1} g(\rho) d \rho-\frac{t^{2}}{\Gamma(\beta)} \int_{0}^{1}(1-\rho)^{\beta-1} g(\rho) d \rho \\
+\delta t^{2} \int_{0}^{1} h(\rho) d \rho
\end{gathered}
$$

Let $C=\int_{0}^{1} h(\rho) d \rho$, then from the above equality, we can get

$C=\int_{0}^{1} h(t) d t$ 
$=-\frac{1}{\Gamma(\beta)} \int_{0}^{1 t} \int_{0}^{t}(t-\rho)^{\beta-1} g(\rho) d \rho d t+\frac{1}{\Gamma(\beta)} \iint_{0}^{1} t^{2}(1-\rho)^{\beta-1} g(\rho) d \rho d t+\delta \iint_{0}^{1} t^{2} h(\rho) d \rho d t$

$C=-\frac{1}{\beta \Gamma(\beta)} \int_{0}^{t}(1-\rho)^{\beta-1} g(\rho) d \rho-\frac{1}{3 \Gamma(\beta)} \int_{0}^{1}(1-\rho)^{\beta-1} g(\rho) d \rho+\frac{\delta}{3} C$

$\mathrm{C}=-\frac{3}{(3-\delta)} \int_{0}^{1} \frac{(1-\rho)^{\beta}}{\beta \Gamma(\beta)} g(\rho) d \rho+\frac{1}{(3-\delta) \Gamma(\beta)} \int_{0}^{1}(1-\rho)^{\beta-1} g(\rho) d \rho$

Substituting this value in (5), we get the following expression for the function $h$

$$
\begin{gathered}
h(t)=-\frac{1}{\Gamma(\beta)} \int_{0}^{t}(t-\rho)^{\beta-1} g(\rho) d \rho+\frac{t^{2}}{\Gamma(\beta)} \int_{0}^{1}(1-\rho)^{\beta+1} g(\rho) d \rho \\
\quad-\frac{3 \delta t^{2}}{(3-\delta) \Gamma(\beta)} \int_{0}^{1} \frac{(1-\rho)^{\beta}}{\beta} g(\rho) d \rho+\frac{\delta t^{2}}{(3-\delta) \Gamma(\beta)} \int_{0}^{1}(1-\rho)^{\beta-1} g(\rho) d \rho \\
=-\frac{1}{\Gamma(\beta)} \int_{0}^{t}(t-\rho)^{\beta-1} g(\rho) d \rho+\int_{0}^{1} \frac{3 t^{2}(1-\rho)^{\beta-1}(\beta-\delta+\delta \rho)}{\beta(3-\delta) \Gamma(\beta)} g(\rho) d \rho \\
=-\frac{1}{\Gamma(\beta)} \int_{0}^{t}(t-\rho)^{\beta-1} g(\rho) d \rho+\frac{1}{\Gamma(\beta)} \int_{0}^{t} \frac{3 t^{2}(1-\rho)^{\beta-1}(\beta-\delta+\delta \rho)}{\beta(3-\delta)} g(\rho) d \rho+ \\
=\frac{1}{\Gamma(\beta+1)} \int_{0}^{t} \frac{3 t^{2}(1-\rho)^{\beta-1}(\beta-\delta+\delta \rho)}{\beta(3-\delta)} g(\rho) d \rho \\
+\frac{1}{\Gamma(\beta+1)} \int_{t}^{1} \frac{3 t^{2}(1-\rho)^{\beta-1}(\beta-\delta+\delta \rho)}{3-\delta} g(\rho) d \rho \\
=\int_{0}^{1} G(t, \rho) g(\rho) d \rho .
\end{gathered}
$$

The proof is complete.

\section{The Main Result}

Note : let function $t \in[0,1] \rightarrow \int_{0}^{1}|G(t, \rho)| d \rho$ be continuous on $[0,1]$, so it is bounded.

Let $Q=\operatorname{Sup}\left\{\int_{0}^{1}|G(t, \rho)| d \rho, t \in[0,1]\right\}$ 
Banch's fixed point theorem is our first result.

let $C\left([0,1], R^{+}\right)$denotes the Banach space of all continuous functions, on $[0,1]$ in to $R^{+}$with norm $\|h\|_{\infty}=\operatorname{Sup}\{|h(t)|: t \in[0,1]\}$ where $|$.$| is the complete norm on R$.

Theorem 3.1. Assume that

(S1) Let $\eta$ be a constant, then

$|h(t, x)-h(t, y)| \leq \eta|x-y|$ for $t \in[0,1]$ and every $x, y \in R$

if $\eta Q<1$

Then the boundary value problem (1)-(2) has a unique solution.

\section{Proof:}

Let the operator $J: C \rightarrow C$ be defined as :

$J h(t)=\int_{0}^{t} G(t, \rho) k(\rho, h(\rho)) d \rho$, where $G(t, \rho)$ is the Green's function given by (4) points we will show that $J$ is a contraction and from lemma 2.1; the fixed points of $J$ are solutions to (1)-(2).

Assume that $x, y \in C\left([0,1], R^{+}\right)$.Then,for each $t \in[0,1]$, we get

$$
\begin{aligned}
& |J x(t)-J y(t)| \leq \int_{0}^{1}\left|G ( t , \rho ) \left\|k(\rho, x(\rho))-k(\rho, y(\rho))\left|d \rho \leq \eta\|(x-y)\|_{\infty} \int_{0}^{1}\right| G(t, \rho) \mid d \rho\right.\right. \\
& \quad \leq \eta Q\|x-y\|_{\infty}
\end{aligned}
$$

Hence, we have

$\|J x(t)-J y(t)\|_{\infty} \leq M\|x-y\|_{\infty}$

Where $M=\eta Q<1$.

The theorem is proved.

Our second result is based on the Schauder's theorem.

Theorem 3.2 Assume that

(A1) The function $k:[1,0] \times R^{+} \rightarrow R^{+}$is continues.

(A2) There exists $N \in C\left([0,1], R^{+}\right)$and $\emptyset:[0, \infty) \rightarrow[0, \infty)$ continues and non- dressing such that $|k(t, x)| \leq N \varnothing(|x|)$, for $t \in[0,1]$ and each $x \in R^{+}$.

(A3) There exists a constant $L>0$ such that 


$$
\frac{L}{N_{1} \varnothing(L) Q}>1
$$

where $N_{1}=\operatorname{Sup}\{N(r), r \in[0,1]\}$. If the above conditions hold, then the problem(1)-(2) has at least one solution.

\section{Proof:}

Let $C^{*}=\left\{h \in C\left([0,1], R^{+}\right),\|h\|_{\infty} \leq L\right\}$, where $L$ is a constant. Clearly $C^{*}$ is a closed convex subset of $C\left([0,1], R^{+}\right)$,we will show that $J$ satisfies the condition of Schauder's theorems.

The proof will be in four steps.

Step1: First we show that $J$ is continuous.

Let $\left\{h_{m}\right\}$ be a sequence such that $h_{m} \rightarrow h$ in $C\left([0,1], R^{+}\right)$.Then for each $t \in[0,1]$. We claim that $f$ is continuous

$\left|J h_{m}(t)-J h(t)\right| \leq \int_{0}^{1}|G(t, \rho)|\left|k\left(\rho, h_{m}(\rho)\right)-k(\rho, h(\rho))\right| d \rho$

Since $k$ is continuous $\left\|J h_{m}(t)-J h(\rho)\right\|_{\infty} \rightarrow 0$ as $m \rightarrow \infty$.

Step 2: $J$ map $C^{*}$ into an equicontinuous sets of $C\left([0,1], R^{+}\right)$. Let $h \in C^{*}, t_{1}, t_{2} \in[0,1], t_{1}-t_{2}<$ 0 ,then

$$
\begin{gathered}
\left|J h\left(t_{2}\right)-J h\left(t_{1}\right)\right|=\left|\int_{0}^{1} G\left(t_{2}, \rho\right) k(\rho \cdot h(\rho)) d \rho-G\left(t_{1}, \rho\right) k(\rho \cdot h(\rho)) d \rho\right| \\
\leq \int_{0}^{1}\left|G\left(t_{2}, \rho\right)-G\left(t_{1}, \rho\right)\right||k(\rho \cdot h(\rho))| d \rho \\
\leq N_{1} \varnothing(L) \int_{0}^{1}\left|G\left(t_{2}, \rho\right)-G\left(t_{1}, \rho\right)\right| d \rho
\end{gathered}
$$

As $t_{1} \rightarrow t_{2}$ the right hand side tends to zero as $t_{1} \rightarrow t_{2}$.

So the operator $J$ is completely continuous according to Arzela-Ascoli theorem.

Step 3: J maps $C^{*}$ in to a bounded set of $C$. Let $h \in C^{*}$, (A2) then for each $t \in[0,1]$, implies

$$
\begin{aligned}
& |J h(t)| \leq \int_{0}^{1}|G(t, \rho)||k(\rho, h(\rho))| d \rho \\
& \leq N_{1} \emptyset\left(\|h\|_{\infty}\right) \int_{0}^{1}|G(t, \rho)| d \rho
\end{aligned}
$$

Therefore

$\|J h(t)\|_{\infty} \leq N \varnothing(L) Q:=s$.

Step4: $J\left(C^{*}\right) \subset C^{*}$ 
Let $h \in C^{*}$, then $J h(t) \in C^{*}$. For each $t \in[0,1]$,we get

$|J h(t)| \leq \int_{0}^{1}|G(t, \rho)||k(\rho . h(\rho))| d \rho \leq N_{1} \varnothing\left(\|h\|_{\infty}\right) \int_{0}^{1}|G(t, \rho)| d \rho$

thus $\|J h\|_{\infty} \leq N_{1} \varnothing(L) Q$

By (7), we have

$\|J h(t)\|_{\infty} \leq L$

so, the operator $J$ has a fixed point $h$ which will be a solution for (1)-(2).

\section{Conclusion}

In this paper, we study boundary value problem with integral conditions for fractional differential equation involving Caputo derivative of order $\beta \in(3,4)$. We establish the existence and uniqueness results of the solution. Our first result is based on Banach contraction principle and our second result is based on Schauder's fixed point theorem.

\section{References}

[1] Kilbas, A.A. Srivastava, H.M. and Trujillo, J.J. "Theory and Applications of Fractional Differential Equations", North-Holland Mathematical studies,Elsevier science,B.V.Amsterdam the Nether lands,vol.204,2006.

[2] Lakshmikantham,V. Leela, S. L. and Devi, J.V. " Theory of Fractional Dynamic Systems", Cambridge Scientific Publishers,2009.

[3] Miller, K.S. and Ross, B. "An introduction to the Fractional Calculus and Fractional Differential Equations", John Wiley, New york, 1993.

[4] Ahmad, B. and Alsaedi, A. and Alghamdi, B.S.,Nonlinear Analysis, Real worldApplications,vol.9,no.4, pp.(1727-1740),2008.

[5] Xunhuan, W. Liping,W. and Qinghong, Z.,Journal of Nonlinear Science and Applications,8,pp(309-314)2015.

[6] Youyu, W,Shuilian,I. and Qichao,W."boundary Value Problems",4,2018.

[7] Mohammed, A.M. Mohammed, S.A. and Satish,R.P.,General Mathmatics,pp(1-10),12Oct.2019.

[8] Podlubny, I."Fractional Differential Equations", Mathematical in science and Engineering,Academic Press,San Diego, Calif USA,vol.198,1999. 
Journal of Education and Science (ISSN 1812-125X), Vol: 29, No: 3, 2020 (237-245)

[9] Granas, A. and Dugundji, J."Fiexd point theory",Springer-verlag,New York,2003. 